\title{
Special Section on Al-Empowered Internet of Things for Smart Cities
}

One of the key enablers for smart cities is the Internet of Things (IoT), which can exploit stateof-the-art communication technologies to support advanced services. However, IoT devices and management systems are typically manufactured by multiple vendors with multiple processes and standards. Furthermore, these devices will generate large amounts of data from different sources and types of sensors, which cannot be effectively processed by traditional methods. In addition, the unstructured data in IoT plays an important role for building smart cities, whereas transmitting and processing these unstructured data consume substantial energy. Therefore, the data transmission and processing in IoT for smart cities should be performed in a more intelligent manner. Recently, artificial intelligence (AI) has emerged as a powerful weapon that supports very efficient data analysis and makes accurate decisions on service provisions of various kinds. Combining IoT with advanced AI technology can make the city smarter. AI-empowered solutions, such as deep learning and reinforcement learning, can better process the vast amounts of real-time data that stream from IoT devices to support intelligent services for smart cities. In light of this potential, this special section provides a venue to comprehensively cover algorithms, frameworks, technologies, and applications of AI-empowered IoT for smart cities. After a strict peer review, 10 papers were selected for publication in this special issue. Details of these selected papers are as follows.

"Power Side-Channel Analysis of RNS GLV ECC Using Machine and Deep Learning Algorithms" by Mehrabi et al. proposes an RNS (Residue Number system) GLV (Gallant Lambert Vanstone) elliptic curve cryptography core that is immune to machine-learning- and deep-learning-based sidechannel attacks. The experimental analysis confirms that the proposed crypto core does not leak any information about the private key, and therefore it is suitable for hardware implementations.

"ISDNet: AI-Enabled Instance Segmentation of Aerial Scenes for Smart Cities" by Garg et al. proposes ISDNet (Instance Segmentation and Detection Network), a novel network to perform instance segmentation and object detection on visual data captured by UAVs. This work enables aerial image analytics for various needs in a smart city. ISDNet makes use of effective anchors to accommodate varying object scales and sizes. The proposed method obtains state-of-the-art results in the aerial context.

In "Robust Facial Image Super-Resolution by Kernel Locality-Constrained Coupled-Layer Regression," Gao et al. design a robust context-patch facial image super-resolution scheme via a KLC2LR (Kernel Locality-constrained Coupled-layer Regression) scheme to obtain the desired high-resolution version from the acquired low-resolution image. The compared experiments in the noisy and noiseless cases have verified that the suggested methodology performs better than many existing predominant facial image super-resolution methods.

\section{ACM Reference format:}

Wei Wei, Ammar Rayes, Wei Wang, and Yiduo Mei. 2021. Special Section on AI-Empowered Internet of Things for Smart Cities. ACM Trans. Internet Things 21, 3, Article 64 (May 2021), 3 pages.

https://doi.org/10.1145/3460868

(C) 2021 Copyright held by the owner/author(s).

1533-5399/2021/05-ART64

https://doi.org/10.1145/3460868 
In "Predictive Analytics for Smart Parking: A Deep Learning Approach in Forecasting of IoT Data," Piccialli et al. present and discuss an innovative deep-learning-based ensemble technique in forecasting parking space occupancy to reduce the search time for parking and to optimize the flow of cars in particularly congested areas, with an overall positive impact on traffic in urban centers. The proposed approach has been assessed on a real IoT dataset composed of more than 15 million collected sensor records. Obtained results demonstrate that the proposed approach outperforms both single predictors and the widely used strategy of the mean providing inherently robust predictions.

“Deep Attentive Multimodal Network Representation Learning for Social Media Images” by Huang et al. proposes a DAMGE (Deep Attentive Multimodal Graph Embedding) model for more effective social image representation learning. It also introduces both small- and large-scale datasets to conduct extensive experiments, of which the results confirm the superiority of the proposal on the tasks of social image classification and link prediction.

In "Random Graph-Based Multiple Instance Learning for Structured IoT Smart City Applications," Chiu et al. propose a common random subgraph model, aiming at identifying a subgraph pattern that is the structural "core" that conveys the probabilistically distributed graph characteristics. The experimental results show that the generated common random subgraph is highly expressive in conveying the basic structure and probabilistic properties of the data even in the presence of probabilistic variations among graph components' attributes.

"Machine Learning Empowered IoT for Intelligent Vehicle Location in Smart Cities" by Wan et al. constructs a vehicle localization system architecture composed of multiple IoT with arbitrary array configuration and a large amount of vehicles in smart cities. Simulation outcomes verify the excellent performance of the proposed vehicle number estimation methods and the DOA (Direction of Arrival) estimation method in smart cities, and the vehicle positions can be achieved with high estimation accuracy.

"Spatio-Temporal Bayesian Learning for Mobile Edge Computing Resource Planning in Smart Cities" by Ale et al. proposes a spatio-temporal Bayesian hierarchical learning approach to learn and predict the distribution of mobile edge computing resource demand over space and time to facilitate mobile edge computing deployment and resource management. Finally, the simulated results show that resources allocated based upon the models' predictions are exploited more efficiently than the resources are equally divided into all servers in unobserved areas.

In "Sustainable Security for the Internet of Things Using Artificial Intelligence Architectures," Iwendi et al. present a deep-learning methodology for detecting cyber-attacks on the IoT using a long short-term network (LSTM) classifier. A detailed metric representing the results in tabular form was used to compare how the proposed model was better than other state-of-the-art models in detecting cyber-attacks with proficiency.

In "Multimodal Brain Tumor Segmentation Based on an Intelligent UNET-LSTM Algorithm in Smart Hospitals," Hu et al. propose using multimodal brain tumor images combined with the UNET (U-shaped Network) and LSTM models to construct a new network structure with a mixed loss function to solve sample imbalance and describe an intelligent segmentation process to identify brain tumors. The results of the proposed network compared with those of other brain tumor segmentation methods show that the proposed algorithm can segment different tumor lesions more accurately.

We are extremely grateful to Professor Liu, the Editor-in-Chief of ACM Transactions on Internet Technology, for providing the opportunity to organize this special issue, and for constant and prompt support. We also thank all of the authors for their valuable contributions to this special 
issue, and all of the volunteer reviewers for their hard work in evaluating the submissions and their helpful comments that certainly contributed to the quality of the published articles.

Wei Wei

Xi'an University of Technology

Ammar Rayes

Cisco Systems

Wei Wang

University of Macau

Yiduo Mei

Beijing Jiaotong University 